\title{
GENETIC MODELS OF SEXUAL AND NATURAL SELECTION IN MONOGAMOUS ORGANISMS
}

\author{
P. O'DONALD \\ Deportment of Genetics, University of Cambridge, Downing Street, \\ Cambridge CB2 3EH, England
}

Received 26.xi.79

\begin{abstract}
SUMmary
Darwin's theory of sexual selection in monogamous organisms is described in terms of specific genetic models in which the sexually selected characters are either dominant and recessive or show no dominance in their effects. Mating preferences determine the sexual advantage of particular phenotypes or genotypes. The equilibrium frequencies and eigenvalues giving the rate of approach to equilibrium are derived from recurrence equations of genotypic frequencies in successive generations. When sexual selection alone acts on a population, the ultimate outcome of evolution is identical in both monogamous and polygynous organisms: the same equilibria are usually reached in both mating systems; but polygyny produces a faster rate of selection than mon ogamy. In some of the cases in which there is no dominance of the sexually selected characters, alternative equilibria may also be reached, as well as the single polygynous equilibrium. The advent of natural selection gives rise to different equilibria in monogamous and polygynous organisms. From data of the reproductive success of birds, the average fertility of the preferential matings can be calculated relative to the fertility of the matings that take place at random. From these values of relative fertilities, it can be shown that a sexually selected character will be maintained in balance with natural selection at a lower equilibrium frequency in a monogamous species than in a polygynous species. Observations show that sexual dimorphisms are more highly developed in polygynous organisms, thus supporting hypotheses of $D$ arwin and Fisher that characters for sexual competition evolve until a point of balance is reached between natural and sexual selection.
\end{abstract}

\section{INTRODUCTION}

IN the Descent of Man, Darwin (1871) put forward a subtle and general theory to explain how sexual selection would take place in monogamous organisms. In Darwin's words, the theory was stated as follows:

"Let us take any species, a bird for instance, and divide the females inhabiting a district into two equal bodies, the one consisting of the more vigorous and better-nourished individuals, and the other of the less vigorous and healthy. The former, there can be little doubt, would be ready to breed in the spring before the others; and this is the opinion of Mr. Jenner Weir, who has carefully attended to the habits of birds during many years. There can also be no doubt that the most vigorous, best-nourished and earliest breeders would on an average succeed in rearing the largest number of fine offspring. The males, as we have seen, are generally ready to breed before the females; the strongest, and with 
some species the best armed of the males, drive away the weaker; and the former would then unite with the more vigorous and better-nourished females, because they are the first to breed. Such vigorous pairs would surely rear a larger number of offspring than the retarded females, which would be compelled to unite with the conquered and less powerful males, supposing the sexes to be numerically equal; and this is all that is wanted to add, in the course of successive generations, to the size, strength and courage of the males, or to improve their weapons."

Darwin also pointed out that the advantage gained by the earlier breeders would also lead to sexual selection by female preference; for

" the more vigorous females, which are the first to breed, will have the choice of many males; and though they may not always select the strongest or best armed, they will select those which are vigorous and well armed, and in other respects the most attractive. Both sexes, therefore, of such early pairs would as above explained, have an advantage over others in rearing offspring; and this apparently has sufficed during a long course of generations to add not only to the strength and fighting powers of the males, but likewise to their various ornaments or other attractions."

The correlation of earlier breeding date with greater breeding success that Darwin postulated is now well established for many birds. O'Donald (1972b, 1973a, 1974) set up computer models of sexual selection in monogamous birds, using the empirical relationship of breeding date and breeding success found in a seabird, the Arctic Skua (O'Donald, 1972a). This bird is polymorphic with three phenotypes in its plumage: a non-melanic, pale phenotype; and two melanic phenotypes, intermediate and dark. The distributions of the breeding dates of the three phenotypes have been fitted to models of female preference for the intermediates and darks (O'Donald, 1976, 1979; O'Donald and Davis, 1977). Selection takes place exactly as Darwin had postulated.

Given estimates of the female preferences, the computer models can be used to determine the effects of selection. In the models, the breeding season is divided into successive intervals. The breeding success in each interval is given by the average number of chicks fledged by each pair. The matings that take place are determined by the choices of the females: as Darwin suggested, preferred phenotypes tend to be chosen as mates in the earlier intervals and thus gain an advantage; the greater the proportion of females with preferences, the earlier the matings of the preferred phenotypes and the greater their selective advantage. The selective values thus produced are frequency-dependent and may produce stable polymorphisms if more than one phenotype is preferred (O'Donald, 1974, 1976, 1979). This monogamous sexual selection always produces the same equilibria as polygynous sexual selection. The actual values of the breeding success of pairs mating in each of the different intervals of the breeding season has no effect on the final equilibrium frequency attained. Various actual and hypothetical distributions of breeding success in relation to breeding date all give the same equilibrium frequency. As we should expect, however, a greater difference in breeding success between early and late pairs produces a faster rate of approach to the equilibrium. 
Two general models of mating preferences were programmed for computer simulation. In one model, females may express separate preferences for each of the three genotypes determined by two alleles at a locus. For example, dark and pale Arctic Skuas represent the two different homozygotes; intermediates represent the heterozygotes. In the other model, females may prefer either a dominant or a recessive phenotype. If $\alpha, \beta$ and $\gamma$ are the proportions of females with separate preferences for males with $A A, A a$ and $a a$ genotypes, then the allele $A$ reaches equilibrium at a gene frequency

$$
p_{*}=\left(\alpha+\frac{1}{2} \beta\right) /(\alpha+\beta+\gamma)
$$

This was shown mathematically for models of polygynous selection (O'Donald, 1973b). But it also appears to hold with monogamous matings for a wide range of numerical values of $\alpha, \beta$ and $\gamma$. If $\alpha$ and $\beta$ are the preferences for the dominant and recessive phenotypes, $A$ and $a$, an equilibrium is always reached at a frequency of $a$ given by

$$
w_{*}=\beta /(\alpha+\beta)
$$

The gene frequency of the allele is then given by

$$
q_{*}=\sqrt{ } w_{*}
$$

The computer model of monogamous sexual selection has been formulated in terms of the general breeding ecology of birds. Simulation is used to determine the frequencies of matings between each genotype in each interval of the breeding season. The numbers of offspring produced by each of the different matings are then calculated from actual or hypothetical values of breeding success. It seems hardly possible to describe this complicated model in terms of explicit differential or finite difference equations. For mathematical analysis, a simpler model of monogamous sexual selection is required. But a simpler model need not be biologically unrealistic. As a result of the complicated sequence of events taking place during the breeding season, the preferential matings take place sooner on average and produce more offspring than the random matings. The model can thus be simplified by assuming that all the preferential matings take place first at one level of fertility, followed by the random matings at another, lower level of fertility. In this paper, I analyse the effects of three different genetic modes of expression of mating preferences: (i) females may prefer phenotypes $A$ or $a$, of which $A$ is dominant to $a$; (ii) females may prefer genotypes $A A, A a$ or $a a$; (iii) females may prefer a dominant or recessive phenotype which reduces the chances of survival, natural selection thus opposing sexual selection. Natural selection must often have been in opposition to sexual selection in the evolution of characters for sexual display: the red throat of the stickleback, which attracts the female, also attracts predators (see O'Donald, 1979); the tail of the peacock must hinder escape.

\section{Preferences for two phenotypes With dominance}

The two phenotypes, $A$ and $a$, occur in a population at frequencies $1-w$ and $w$. They are preferred by proportions $\alpha$ and $\beta$ of the females. $A$ is dominant, consisting of the genotypes $A A$ and $A a$ at frequencies $u$ and $v$; $a$ is recessive, consisting of the genotype aa. Since, in monogamy, males who have found a mate do not mate again (they are sampled "without 
replacement" by the females!), there may be too few males of one phenotype to satisfy all the females that prefer them. Some of the females will thus be unable to mate according to their preferences. I assume these females mate at random with any of the males that are left, like the females who have no preferences for particular males. Three cases must be considered: Case (i), in which $\alpha \leqq 1-w, \beta \leqq w$, so that all females with preferences can mate with the males they prefer; Case (ii), in which $\alpha \leqq$ $1-w, \beta>w$, so that too many females prefer $a$ males; and Case (iii), in which $\alpha>1-w, \beta \leqq w$, so that too many females prefer $A$ males.

Case (i). Assume that the conditions for Case (i) hold. Males then take part in matings with their genotypes at frequencies:

\begin{tabular}{ccc} 
& in preferential & \multicolumn{1}{c}{ in random } \\
matings & matings \\
$A A$ & $\alpha u /(1-w)$ & $u-\alpha u /(1-w)$ \\
$A a$ & $\alpha v /(1-w)$ & $v-\alpha v /(1-w)$ \\
$a a$ & $\beta$ & $w-\beta$
\end{tabular}

Matings between different genotypes occur with frequencies and fertilities shown in table 1 . It has been assumed that the random matings have a fertility $f(1 \geqq f \geqq 0)$ compared with the fertility $1 \cdot 0$ of the preferential

TABLE 1

Frequencies of preferential and random matings and the fertilities of matings as determined by preferences for both dominant and recessive phenotypes

(i) Frequencies of matings

$\begin{array}{lll}\text { Mating } & \begin{array}{l}\text { Preferential component } \\ \text { of frequency }\end{array} & \begin{array}{l}\text { Random component } \\ \text { of frequency }\end{array} \\ A A \times A A & \alpha u^{2} /(1-w) & u^{2}-\alpha u^{2} /(1-w) \\ A A \times A a & 2 \alpha u v /(1-w) & 2 u v-2 \alpha u v /(1-w) \\ A A \times a a & \beta u+\alpha u w /(1-w) & 2 u w-\beta u-\alpha u w /(1-w) \\ A a \times A a & \alpha v^{2} /(1-w) & v^{2}-\alpha v^{2} /(1-w) \\ A a \times a a & \beta v+\alpha v w /(1-w) & 2 v w-\beta v-\alpha v w /(1-w) \\ a a \times a a & \beta w & w^{2}-\beta w\end{array}$

(ii) Fertilities of matings

$\begin{array}{ll}\text { Matings } & \text { Fertility } \\ A A \times A A & {\left[f u^{2}+\alpha(1-f) u^{2} /(1-w)\right] / T} \\ A A \times A a & {[2 f u v+2 \alpha(1-f) u v /(1-w)] / T} \\ A A \times a a & {[2 f u w+\alpha(1-f) u w /(1-w)+\beta(1-f) u] / T} \\ A a \times A a & {\left[f v^{2}+\alpha(1-f) v^{2} /(1-w)\right] / T} \\ A a \times a a & {[2 f v w+\alpha(1-f) v w /(1-w)+\beta(1-f) v] / T} \\ a a \times a a & {\left[f w w^{2}+\beta(1-f) w\right] / T} \\ & T=f+(1-f)(\alpha+\beta)=1-(1-f)(1-\alpha-\beta)\end{array}$

matings. In the next generation, the genotypic frequencies become the following:

$$
\begin{aligned}
T u^{\prime} & =f p^{2}+\alpha(1-f) p^{2} /(1-w) \\
T v^{\prime} & =2 f p q+\alpha(1-f)(2 p q-p w) /(1-w)+\beta(1-f) p \\
T w^{\prime} & =f q^{2}+\alpha(1-f)\left(q^{2}-q w\right) /(1-w)+\beta(1-f) q
\end{aligned}
$$

where $T=f+(1-f)(\alpha+\beta)$ as shown in the table. It is obvious, as we should expect, that when $f=1$ the relative fertilities simply equal the 
frequencies of random mating and the genotypic frequencies follow the Hardy-Weinberg Law. In terms of the gene frequencies $p^{\prime}=u^{\prime}+\frac{1}{2} v^{\prime}$, $p=u+\frac{1}{2} v$ and $q=1-p$, we obtain

and hence

$$
T p^{\prime}=f p+\alpha p(1-f)\left(1-\frac{1}{2} w\right) /(1-w)+\frac{1}{2} \beta p(1-f)
$$

$$
\begin{aligned}
T \Delta p & =T p^{\prime}-T p \\
& =\frac{1}{2} p(1-f)[w(\alpha+\beta)-\beta] /(1-w)
\end{aligned}
$$

showing that at equilibrium when $\Delta p=0, a$ is maintained at the frequency

$$
w_{*}=\beta /(\alpha+\beta)
$$

This equilibrium had previously been obtained mathematically for models of polygynous sexual selection and had also been obtained numerically by computer simulation of monogamous sexual selection. Hardy-Weinberg ratios hold at equilibrium: $u_{*}=p_{*}^{2} ; v_{*}=2 p_{*} q_{*} ;$ and $w_{*}=q_{*}^{2}$.

To find the stability of this equilibrium and the rate of approach to it if stable, it is convenient to consider the pair of recurrence equations

$$
\begin{aligned}
T p^{\prime} & =f p+\frac{1}{2} \beta p(1-f)+\alpha p(1-f)\left(1-\frac{1}{2} w\right) /(1-w) \\
T w^{\prime} & =f q^{2}+\beta q(1-f)+\alpha q(1-f)(q-w) /(1-w)
\end{aligned}
$$

and obtain the gradient matrix

$$
\left[\begin{array}{ll}
\partial p^{\prime} / \partial p_{*} & \partial p^{\prime} / \partial w_{*} \\
\partial w^{\prime} / \partial p_{*} & \partial w^{\prime} / \partial w_{*}
\end{array}\right]
$$

in which the partial derivatives with respect to $p$ and $w$ are evaluated at the points $p_{*}$ and $w_{*}$. Carrying out this differentiation, we find that the gradient matrix takes the general form (O'Donald, 1980, Heredity, this issue, p. 312)

where, for the present case,

$$
\left[\begin{array}{ll}
1 & \frac{1}{2} \theta / q_{*} \\
-2 q_{*} & -\theta
\end{array}\right]
$$

$$
\theta=\frac{p_{*} q_{*}(1-f)(\alpha+\beta)^{2}}{\alpha[f+(1-f)(\alpha+\beta)]}
$$

The non-zero eigenvalue of this matrix, $\lambda=1-\theta$, shows that for all positive values of $\alpha$ and $\beta \lambda<1$ and $p_{n}$ converges to its equilibrium value $p_{*}$ at a geometric rate $\lambda$ : if $p_{0}$ is a small deviation from $p_{*}$, then approximately after $n$ generations

$$
p_{n}-p_{*}=\lambda^{n}\left(p_{0}-p_{*}\right)
$$

Case (ii). According to the conditions for Case (ii), $\alpha \leqq 1-w, \beta>w$, so that too few $a$ males are available to mate with all the females that prefer them. The males mate with the frequencies:

\begin{tabular}{ccc} 
& in preferential & \multicolumn{1}{c}{ in random } \\
matings & matings \\
$A A$ & $\alpha u /(1-w)$ & $u-\alpha u /(1-w)$ \\
$A a$ & $\alpha v /(1-w)$ & $v-\alpha v /(1-w)$ \\
$a a$ & $w$ & 0
\end{tabular}


giving the recurrence equations

$$
\begin{aligned}
T u^{\prime} & =p^{2}-p^{2}(1-f)(1-\alpha-w) /(1-w) \\
T v^{\prime} & =2 p q-(1-f)(2 p q-p w)(1-\alpha-w) /(1-w) \\
T w^{\prime} & =q^{2}-(1-f)\left(q^{2}-q w\right)(1-\alpha-w) /(1-w)
\end{aligned}
$$

so that

$$
T p^{\prime}=p-p(1-f)\left(1-\frac{1}{2} w\right)(1-\alpha-w) /(1-w)
$$

and

where

$$
T \Delta p=-\frac{1}{2} p w(1-f)(1-\alpha-w) /(1-w)
$$

$$
T=f+(1-f)(\alpha+w)=1-(1-f)(1-\alpha-w) .
$$

Thus equilibrium is apparently reached when

$$
w_{*}=1-\alpha
$$

But, by the conditions for this case, $\beta>w$, and hence at equilibrium $\beta>1-\alpha$ so that we should have

$$
\alpha+\beta>1
$$

which contradicts the essential premise of the model that $\alpha+\beta \leqq 1$. The frequency $w_{*}=1-\alpha$ cannot therefore represent the equilibrium point. However, the equation for $\Delta p$ shows that near fixation of $A, \Delta p<0$ : the allele $a$ can enter the population and increase in frequency. Near fixation of $a, A$ can enter the population. As a result of selection, the gene frequency changes so as to ensure that ultimately the conditions of Case (i) are realised. Since, at the start of selection, either $A$ or $a$ must be near to fixation, the conditions for either Case (ii) or Case (iii) must initially hold: when $A$ is near to fixation, $w$ is small and the conditions for Case (ii) must hold; similarly, when $a$ is near to fixation, the conditions for Case (iii) must hold. If $A$ is near to fixation, then $\Delta p<0$ for all values $w \leqq 1-\alpha$. Therefore $a$ increases in frequency until the conditions for Case (ii) become those for Case (i), hence giving the equilibrium

$$
w_{*}=\beta /(\alpha+\beta)
$$

Case (iii). The conditions $\alpha>1-w, \beta \leqq w$ show that too few $A$ males are available to mate with all the females that prefer them. The males mate with frequencies:

$\begin{array}{ccc} & \text { in preferential } & \text { in random } \\ & \text { matings } & \text { matings } \\ A A & u & 0 \\ A a & v & 0 \\ a a & \beta & w-\beta\end{array}$

giving the recurrence equations

$$
\begin{aligned}
T u^{\prime} & =p^{2} \\
T v^{\prime} & =2 p q-p(1-f)(w-\beta) \\
T w^{\prime} & =q^{2}-q(1-f)(w-\beta)
\end{aligned}
$$


and

so that

$$
\mathcal{T} p^{\prime}=p-\frac{1}{2} p(1-f)(w-\beta)
$$

$$
T \Delta p=\frac{1}{2} p(1-f)(w-\beta) .
$$

This would also entail an impossible equilibrium, like Case (ii), giving rise to the contradiction

$$
\alpha+\beta>1
$$

In the same way as the conditions for Case (ii) imply that $A$ is initially near fixation, so also conditions for Case (iii) imply that $a$ is near fixation. The equation for $\Delta p$ shows that $\Delta p>0$ for all values $w \geqq \beta$. But before the point $w=\beta$ can be attained, Case (iii) becomes converted to Case (i) and equilibrium is reached at the point

$$
w_{*}=\beta /(\alpha+\beta)
$$

The results of sexual selection in monogamous and polygynous organisms can now be compared. Obviously, if all matings have the same fertility, monogamy always gives rise to random mating as a whole, regardless of the mating preferences that may have been expressed. The genotypic frequencies immediately follow the Hardy-Weinberg Law, so that with initial frequencies $p_{0}$ and $q_{0}$ then at equilibrium

$$
w_{*}=q_{0}^{2}
$$

But if later random matings have reduced fertility compared to earlier preferential matings, then the equilibrium attained is always

$$
w_{*}=\beta /(\alpha+\beta)
$$

exactly as when matings are polygynous. In all cases at this equilibrium, the genotypic frequencies follow that Hardy-Weinberg Law, so that

$$
q_{*}=\sqrt{\beta /(\alpha+\beta)}
$$

Of course, this equilibrium may be approached very slowly if the reduction

\begin{tabular}{|c|c|c|c|}
\hline Model & & $\begin{array}{l}\text { Equilibrium } \\
\text { frequency }\end{array}$ & $\begin{array}{l}\text { Geometric rate of } \\
\text { approach to equilibrium }\end{array}$ \\
\hline \multirow{2}{*}{$\begin{array}{l}\text { Equal fertility } \\
\text { for all matings }\end{array}$} & Monogamy & $w_{*}=q_{0}^{2}$ & $\lambda=1$ \\
\hline & Polygyny & $w_{*}=\beta /(\alpha+\beta)$ & $\lambda=1-p_{*} q_{*}(\alpha+\beta)^{2} / \alpha$ \\
\hline \multirow{2}{*}{$\begin{array}{l}\text { Fertility } \\
\text { reduced in } \\
\text { later matings }\end{array}$} & Monogamy & $w_{*}=\beta /(\alpha+\beta)$ & $\lambda=1-\frac{p_{*} q_{*}(1-f)(\alpha+\beta)^{2}}{\alpha[f+(1-f)(\alpha+\beta)]}$ \\
\hline & Polygyny & $w_{*}=\beta /(\alpha+\beta)$ & $\lambda=1-\frac{p_{*} q_{*}(\alpha+\beta)^{2}}{\alpha[f+(1-f)(\alpha+\beta)]}$ \\
\hline
\end{tabular}
in fertility is only slight. Polygyny always produces a faster rate of approach than monogamy. These results are summarised in table 2. For purposes of comparison, results are also given for the case in which random matings

TABLE 2

Equilibrium frequencies and rates of approach to equilibrium in models with dominance and either polygynous or monogamous mating 
suffer reduced fertility in polygynous species. In the extreme case, when $f=0$, we have

$$
\lambda=1-p_{*} q_{*}(\alpha+\beta) / \alpha
$$

for both monogamous and polygynous matings. This represents the fastest rate at which sexual selection can occur. Generally, for values $1>f>0$, sexual selection will be faster with polygyny. It should be noted that if $f>1$, then $\lambda>1$; the equilibria are still the same but are unstable.

\section{SEPARATE PREFERENCES FOR EACH OF THREE GENOTYPES}

When females may prefer one of three genotypes, three parameters, $\alpha, \beta$ and $\gamma$, are required for the proportions of females who prefer $A A, A a$ or aa males. There are now seven possible sets of initial conditions:

$$
\begin{aligned}
& \text { Case (i), } \alpha \leqq u, \beta \leqq v, \gamma \leqq w ; \\
& \text { Case (ii), } \alpha \leqq u, \beta \leqq v, \gamma>w ; \\
& \text { Case (iii), } \alpha \leqq u, \beta>v, \gamma \leqq w ; \\
& \text { Case (iv), } \alpha>u, \beta \leqq v, \gamma \leqq w ; \\
& \text { Case (v), } \alpha \leqq u, \beta>v, \gamma>w ; \\
& \text { Case (vi), } \alpha>u, \beta \leqq v, \gamma>w ; \\
& \text { Case (vii), } \alpha>u, \beta>v, \gamma \leqq w .
\end{aligned}
$$

Cases (ii) and (iv) and (v) and (vii) should give essentially similar results in view of their symmetries.

Case $(i)$. All females with preferences can mate with the males they prefer. The males therefore mate with the frequencies:

$\begin{array}{ccc} & \begin{array}{c}\text { in preferential } \\ \text { matings }\end{array} & \text { in random } \\ \text { matings } \\ A A & \alpha & u-\alpha \\ A a & \beta & v-\beta \\ a a & \gamma & w-\gamma\end{array}$

The matings then take place with fertilities as shown in table 3. We can see immediately that if $f=1$, the fertilities simply equal the frequencies

TABLE 3

Fertilities of matings with separate preferences for each genotype

Mating
$A A \times A A$
$A A \times A a$
$A A \times a a$
$A a \times A a$
$A a \times a a$
$a a \times a a$

Fertility

$$
\begin{aligned}
& \alpha u+f u(u-\alpha)=u^{2}-u(1-f)(u-\alpha) \\
& \alpha v+\beta u+f v(u-\alpha)+f u(v-\beta)=2 u v-(1-f)(2 u v-\alpha v-\beta u) \\
& \alpha w-\gamma u+f w(u-\alpha)+f u(w-\gamma)=2 u w-(1-f)(2 u w-\alpha w-\gamma u) \\
& \beta v+f v(v-\beta)=v^{2}-v(1-f)(v-\beta) \\
& \beta w+\gamma v+f w(v-\beta)+f v(w-\gamma)=2 v w-(1-f)(2 v w-\beta w-\gamma v) \\
& \gamma w+f w(w-\gamma)=w^{2}-w(1-f)(w-\gamma)
\end{aligned}
$$

In this table, the fertilities must be divided by the factor

$$
T=1-(1-f)(1-\alpha-\beta-\gamma)
$$

to bring the total fertility to $1 \cdot 0$. 
of random mating. The recurrence equations for the genotypes are quickly obtained and hence the recurrence equation for the gene frequency

$$
T p^{\prime}=p-(1-f)\left[p\left(1-\frac{1}{2} \alpha-\frac{1}{2} \beta-\frac{1}{2} \gamma\right)-\frac{1}{2} \alpha-\frac{1}{4} \beta\right]
$$

This is a simple linear equation in one variable. It gives the difference equation

$$
T \Delta p=\frac{1}{2}(1-f)\left[\alpha+\frac{1}{2} \beta-p(\alpha+\beta+\gamma)\right]
$$

showing that

$$
p_{*}=\left(\alpha+\frac{1}{2} \beta\right) /(\alpha+\beta+\gamma)
$$

The Hardy-Weinberg Law holds at this equilibrium, exactly as when matings are polygynous.

If we put $\alpha+\frac{1}{2} \beta=\phi$ and $\alpha+\beta+\gamma=\theta$, then the recurrence equation can be written in the form

$$
p^{\prime}=p\left[\frac{f+\frac{1}{2} \theta(1-f)}{f+\theta(1-f)}\right]+\frac{\frac{1}{2} \phi(1-f)}{f+\theta(1-f)}
$$

with the general solution for all values of $p$

$$
p_{n}-p_{*}=\left(p_{0}-p_{*}\right)\left[\frac{f+\frac{1}{2} \theta(1-f)}{f+\theta(1-f)}\right]^{n} \text {. }
$$

In the polygynous model, the corresponding equation is

$$
p_{n}-p_{*}=\left(p_{0}-p_{*}\right)\left(1-\frac{1}{2} \theta\right)^{n}
$$

As in the model with dominance, the equilibrium is stable for all values $f<1$. The results of selection in monogamous and polygynous species are compared in table 4 .

\section{TABLE 4}

Equilibrium frequencies and rates of approach to equilibrium in models with separate preferences for each genotype and either monogamous or polygynous mating

Model

Equal fertilities

for all matings

Fertility

reduced in

later matings

$$
\begin{aligned}
& \text { Equilibrium } \\
& \text { frequency }
\end{aligned}
$$

$$
p_{*}=p_{0}
$$$$
p_{*}=\phi / \theta
$$$$
p_{*}=\phi / \theta
$$$$
p_{*}=\phi / \theta
$$

Geometric rate of approach to equilibrium

$$
\begin{aligned}
& \lambda=1 \\
& \lambda=1-\frac{1}{2} \theta \\
& \lambda=\frac{f+\frac{1}{2} \theta(1-f)}{f+\theta(1-f)} \\
& \lambda=\frac{f+\frac{1}{2} \theta(1-2 f)}{f+\theta(1-f)}
\end{aligned}
$$

In this table $\phi=\alpha+\frac{1}{2} \beta$ and $\theta=\alpha+\beta+\gamma$.

When a gene is increasing in frequency in a population from a low initial frequency, the conditions will be those for Cases (v) or (vii). For Case (v), the conditions $\alpha \leqq u, \beta>v$ and $\gamma>w$ imply that $a$ is the rare allele, perhaps just having arisen by mutation. As it increases in frequency, the frequency of the genotype $A a$ will show a corresponding increase at first; but, as $a$ 
becomes more common and $a a$ homozygotes start to appear in the population, $A a$ 's increase will slow down. Case (v) may thus become Case (ii) or (iii).

Case $(v)$. Males mate with frequencies:

$\begin{array}{ccc} & \text { in preferential } & \text { in random } \\ \text { matings } & \text { matings } \\ A A & \alpha & u-\alpha \\ A a & v & 0 \\ a a & w & 0\end{array}$

giving the recurrence equation

where

$$
T p^{\prime}=f p+\frac{1}{2}(1-f)\left[\alpha+\frac{1}{2} v+p(\alpha+v+w)\right]
$$

$$
T=f+(1-f)(\alpha+v+w)
$$

and hence the difference equation

$$
T \Delta p=\frac{1}{2}(1-f)\left[\alpha+\frac{1}{2} v-p(\alpha+v+w)\right]
$$

howing that an equilibrium appears to exist at the gene frequency

$$
p_{*}=\left(\alpha+\frac{1}{2} v_{*}\right) /\left(\alpha+v_{*}+w_{*}\right) \text {. }
$$

It is easily shown that the Hardy-Weinberg ratios are maintained at this equilibrium point. Then, since

therefore at equilibrium

$$
\begin{aligned}
q_{*} & =\left(w_{*}+\frac{1}{2} v_{*}\right) /\left(\alpha+v_{*}+w_{*}\right) \\
& =q_{*} /\left(\alpha+v_{*}+w_{*}\right)
\end{aligned}
$$

or

$$
\alpha+v_{*}+w_{*}=1
$$

$$
\alpha=u_{*} .
$$

But since a condition for Case (v) is $\beta+\gamma>v+w$, therefore at the equilibrium $\alpha=u_{*}$, the condition would become $\alpha+\beta+\gamma>1$, which contradicts the essential premise $\alpha+\beta+\gamma \leqq 1$.

The recurrence equation for Case (v) shows that for all $p>p_{*}, p^{\prime}<p$. Fixation of $A$ is therefore an unstable point: if $a$ has entered the population at a low frequency, it will spread. But before the apparent equilibrium of Case ( $v$ ) can be attained, the conditions must change. Case ( $v$ ) is most likely to change into Case (ii): the genotype $A a$ increases in frequency with the increase of $a$, and this may lead to $v>\beta$ as for Case (ii). However, a maximum frequency is attained at $v=\frac{1}{2}$. Therefore, if $\beta>\frac{1}{2}$, the condition $v>\beta$ can never be satisfied. Case (v) would then presumably change into Case (iii).

Case (ii). This case, and also the corresponding Case (iv), cannot be analysed without ambiguity. Males mate with frequencies:

$\begin{array}{ccc} & \begin{array}{c}\text { in preferential } \\ \text { matings }\end{array} & \text { in random } \\ \text { matings } \\ A A & \alpha & u-\alpha \\ A a & \beta & v-\beta \\ a a & w & 0\end{array}$


The difference equation can then be shown to be

$$
T \Delta p=\frac{1}{2}(1-f)\left[\alpha+\frac{1}{2} \beta-p(\alpha+\beta+w)\right]
$$

and hence at equilibrium

$$
p_{*}=\left(\alpha+\frac{1}{2} \beta\right) /\left(\alpha+\beta+w_{*}\right)
$$

Since the Hardy-Weinberg ratios are found at equilibrium,

$$
q_{*}=\left(q_{*}^{2}+\frac{1}{2} \beta\right) /\left(\alpha+\beta+q_{*}^{2}\right)
$$

and the gene frequency of $a$ is given by the solution of the cubic equation

$$
q_{*}^{3}-q_{*}^{2}+(\alpha+\beta) q_{*}-\frac{1}{2} \beta=0 .
$$

This solution does not necessarily contradict the conditions of the model. It may therefore hold until the equilibrium of Case (ii) has actually been attained. However, the difference equation shows that for all $p>p_{*}$, $p^{\prime}<p$, so that fixation of $A$ is an unstable point and $a$ can enter the population. The fixation of $a$ is also an unstable point. Some numerical values of the equilibrium frequency of $a$ are shown in table 5 . When $\alpha \leqq 0 \cdot 2$, the equilibrium frequency contradicts either the condition $\alpha \leqq u$ or the condi-

TABLE 5

Equilibrium frequencies of the allele a when the conditions of Case (ii) hold

$\begin{array}{lllllll}\begin{array}{c}\text { Values } \\ \text { of } \beta\end{array} & 0.01 & 0.05 & 0.1 & \begin{array}{c}0.2 \\ 0.7167 \bullet\end{array} \\ & & & 0.4 & 0.8 \\ 0.01 & 0.9448 \bullet & 0.9419 \bullet & 0.8817 \bullet & \left.\begin{array}{l}0.2560 \\ \mathbf{0 . 0 2 7 2}\end{array}\right\} & \mathbf{0 . 0 1 2 6} & \mathbf{0 . 0 0 6 2} \\ & & & & & \\ 0.05 & 0.9647 \bullet & 0.9209 \bullet & 0.8593 \bullet & 0.6903 \bullet & \mathbf{0 . 0 6 4 1} & \mathbf{0 . 0 3 0 5} \\ 0.1 & 0.9396 \bullet & 0.8948 \bullet & 0.8318 \bullet & 0.6604 \bullet & \mathbf{0 . 1 2 9 0} & \mathbf{0 . 0 5 9 2} \\ 0.2 & 0.8903 * & 0.8442 \bullet & 0.7797 \bullet & 0.6137 \bullet & \mathbf{0 . 2 3 9 2} & - \\ 0.4 & 0.8000 * & 0.7549 * & 0.6947 \bullet & 0.5649 \bullet & \mathbf{0 . 3 4 9 2} & - \\ 0.8 & 0.6766 * & 0.6443 * & 0.6051 * & - & - & -\end{array}$

Roots of the equation for $q_{*}$, other than the values shown, are imaginary. Only when $\alpha=0.2$ and $\beta=0.01$ are three real roots found. Values in bold type indicate a possible equilibrium frequency. The symbol $\bullet$ indicates that at this frequency $\alpha>u_{*}$ which contradicts the condition $\alpha \leqq u$. The symbol $*$ indicates that $\beta>v_{*}$ which contradicts $\beta \leqq v$.

tion $\beta \leqq v$. Larger values of $\alpha$ give rise to no contradictions of these conditions. The value of $\gamma$ must also be greater than $q_{*}^{2}$ for the conditions of Case (ii) to hold. In the table, the values $\alpha=0.2$ and $\beta=0.01$ and all values of $\beta$ with $\alpha=0.4$ and 0.8 give equilibria that agree with the conditions $\alpha \leqq u_{*}, \beta \leqq v_{*}$ and $\alpha+\beta+\gamma \leqq 1$. Given these values, the allele $a$ continues to increase until the equilibrium for Case (ii) is reached without the condition $\gamma>w$ being necessarily violated.

Case (iii). If $\beta$ is a large quantity, it is possible that Case (v) may become Case (iii) in the course of evolution. If so, the males will mate with frequencies:

$\begin{array}{ccc} & \text { in preferential } & \text { in random } \\ \text { matings } & \text { matings } \\ A A & \alpha & u-\alpha \\ A a & v & 0 \\ a a & \gamma & w-\gamma\end{array}$


leading to the following difference equation for the gene frequency

$$
T \Delta p=-\frac{1}{2}(1-f)[u-\alpha-p(1-\alpha-v-\gamma)]
$$

with an equilibrium at

$$
p_{*}=\left(u_{*}-\alpha\right) /\left(1-\alpha-v_{*}-\gamma\right) .
$$

This case is difficult to analyse unless we assume that $\alpha=\gamma$. This assumption gives recurrence equations

Hence

$$
\begin{aligned}
T u^{\prime} & =p^{2}-p(1-f)(u-\alpha) \\
T v^{\prime} & =2 p q-p(1-f)(w-\alpha)-q(1-f)(u-\alpha) \\
T w^{\prime} & =q^{2}-q(1-f)(w-\alpha)
\end{aligned}
$$

so that

$$
\begin{aligned}
\mathcal{T}\left(u^{\prime}-w^{\prime}\right) & =p^{2}-q^{2}-(1-f)(p u-q w-\alpha p+\alpha q) \\
& =p-q-(p-q)(1-f)\left(1-\frac{1}{2} v-\alpha\right)
\end{aligned}
$$

Therefore

$$
u^{\prime}-w^{\prime}=(u-w)\left[\frac{1-(1-f)\left(1-\frac{1}{2} v-\alpha\right)}{1-(1-f)(1-v-2 \alpha)}\right] \text {. }
$$

since

$$
u_{n}-w_{n} \rightarrow 0
$$

$$
\left[\frac{1-(1-f)\left(1-\frac{1}{2} v-\alpha\right)}{1-(1-f)(1-v-2 \alpha)}\right]<1
$$

for all biologically realistic values of $f, v$ and $\alpha$. At equilibrium

$$
\begin{aligned}
& u_{*}=w_{*} \\
& p_{*}=q_{*}=\frac{1}{2}
\end{aligned}
$$

The difference equation when $\alpha=\gamma$ can be written in the form

$$
\Delta p=\frac{-\frac{1}{2}(1-f)[(u-\alpha)-p(1-v-2 \alpha)]}{1-(1-f)(1-v-2 \alpha)} .
$$

This can be differentiated by assuming that Hardy-Weinberg ratios hold approximately, so that

$$
\begin{aligned}
& \frac{d u}{d p}=2 p \\
& \frac{d v}{d p}=2(1-2 p) .
\end{aligned}
$$

Then evaluating the derivative at the point $p=\frac{1}{2}$, we find

$$
\left.\frac{d \Delta p}{d p}\right|_{p=\frac{1}{2}}=-\frac{(1-f)\left(\frac{1}{2}+2 \alpha\right)}{2\left[1-(1-f)\left(\frac{1}{2}-2 \alpha\right)\right]}
$$

The equilibrium is therefore stable and could exist given the conditions $\alpha<\frac{1}{4}, \beta>\frac{1}{2}$ and $\gamma<\frac{1}{4}$. Both fixation states are unstable.

Case (vi). The analysis of Cases (iv) and (vii) is similar to that of Cases (ii) and (v), except that the parameters $\alpha$ and $\gamma$ are interchanged. Only 
Case (vi) remains to be discussed. Clearly the conditions of Case (vi) cannot arise when a newly introduced allele is starting to spread through a population: the conditions $\alpha>u, \gamma>w$ imply that neither $A A$ nor $a a$ are near fixation. Biologically Case (vi) is unrealistic. But it leads to simple, easily analysed recurrence equations as follows:

$$
\begin{aligned}
& T u^{\prime}=p^{2}-\frac{1}{2} p(1-f)(v-\beta) \\
& T v^{\prime}=2 p q-\frac{1}{2}(1-f)(v-\beta) \\
& T w^{\prime}=q^{2}-\frac{1}{2} q(1-f)(v-\beta)
\end{aligned}
$$

Hence we obtain the difference equation

$$
\Delta p=\frac{\frac{1}{2}(1-f)(v-\beta)\left(p-\frac{1}{2}\right)}{1-(1-f)(v-\beta)}
$$

with the two possible equilibria

$$
\begin{aligned}
& v_{*}=\beta \\
& p_{*}=\frac{1}{2}
\end{aligned}
$$

According to the conditions for Case (vi), $\alpha+\gamma>1-v$. Therefore, at the equilibrium $v_{*}=\beta$, we should have $\alpha+\beta+\gamma>1$, which is impossible. At the other equilibrium, the derivative of the expression for $\Delta p$ is given by

$$
\left.\frac{d \Delta p}{d p}\right|_{p=\frac{1}{2}}=\frac{\frac{1}{2}(1-f)\left(\frac{1}{2}-\beta\right)}{1-(1-f)\left(\frac{1}{2}-\beta\right)} .
$$

This equilibrium would only be stable if $\beta>\frac{1}{2}$, contradicting the condition $\beta \leqq v$. The difference equation shows that provided $\beta<v, \Delta p<0$ for all values $p<\frac{1}{2}$. Apparently, therefore, $p<\frac{1}{2}$ defines a domain of attraction towards the fixation of $a$, and similarly $p>\frac{1}{2}$ defines a domain of attraction towards the fixation of $A$. Thus, if the conditions for Case (vi) could hold initially, fixation of one or other allele would seem to follow. However, during the progress to fixation, the initial conditions must change at some point to those for either Cases (ii) or (iv). Both of these cases may then change into Case (i). Ultimately, therefore, an equilibrium will be established according to the conditions either for Cases (ii) or (iv) or for Case (i). This statement is also true if the initial conditions are those for Cases (v) or (vii), which would apply to rare alleles starting to spread through a population.

Separate preferences for each genotype give rise to selection, the outcome of which may thus depend on initial conditions. But the most likely outcome is the establishment of an equilibrium at the gene frequency

$$
p_{*}=\left(\alpha+\frac{1}{2} \beta\right) /(\alpha+\beta+\gamma)
$$

\section{Preference for dominant oR Regessive Phenotypes With NATURAL SELEGTION OF MALES}

Characters that have evolved by sexual selection must often reduce the chances of survival, since an elaborate display to attract the females may also attract predators. The development of heavy plumage and other characters for display may also hinder escape. This natural selection can be 
measured by selective coefficients, $s$ and $t$, where $s$ measures the relative disadvantage of the dominant phenotype and $t$ the relative disadvantage of the recessive. After natural selection against the recessive, the phenotypes $A$ and $a$ occur at frequencies

$$
\begin{aligned}
& {[A]=(1-w) /(1-t w)} \\
& {[a]=w(1-t) /(1-t w)}
\end{aligned}
$$

The single coefficient $t$ is in fact sufficient to measure both advantage and disadvantage if negative values are allowed. Thus, if $t$ is negative, $a$ is advantageous compared to $A$. This is equivalent to using $s$ to measure the disadvantage of $A$, since after selection against $A$

and therefore

$$
\begin{aligned}
{[A] } & =(1-s)(1-w) /[1-s(1-w)] \\
{[a] } & =w /[1-s(1-w)]
\end{aligned}
$$

$$
t=-s /(1-s)
$$

The substitution of $-s /(1-s)$ for $t$ converts selection against the recessive into selection against the dominant.

After natural selection, matings then take place with preferences $\alpha$ for the dominant $A$ or $\beta$ for the recessive $a$.

\section{(i) Preference for a deleterious recessive $(\alpha=0)$}

At the start of selection, conditions for one of two alternative cases must hold: Case (i), in which $\beta \leqq w(1-t) /(1-t w)$; and Case (ii), in which $\beta>w(1-t) /(1-t w)$. If $A$ has recently entered the population at a low

\section{TABLE 6}

Frequencies of preferential and random matings and the fertilities of matings determined by female preferences for both dominant and recessive phenotypes

(i) Frequencies of matings

$\begin{array}{ll}\text { Mating } & \begin{array}{l}\text { Preferential component } \\ \text { of frequency }\end{array} \\ A A \times A A & \alpha u^{2} /(1-w) \\ A A \times A a & 2 \alpha u v /(1-w) \\ A A \times a a & \alpha u w /(1-w)+\beta u \\ A a \times A a & \alpha v^{2} /(1-w) \\ A a \times a a & \alpha v w /(1-w)+\beta v \\ a a \times a a & \beta w\end{array}$

$$
\begin{aligned}
& \text { Random component } \\
& \text { of frequency } \\
& u^{2} /(1-t w)-\alpha u^{2} /(1-w) \\
& 2 u v /(1-t w)-2 \alpha u v /(1-w) \\
& u w(2-t) /(1-t w)-\alpha u w /(1-w)-\beta u \\
& v^{2} /(1-t w)-\alpha v^{2} /(1-w) \\
& v w(2-t) /(1-t w)-\alpha v w /(1-w)-\beta v \\
& w^{2}(1-t) /(1-t w)-\beta w
\end{aligned}
$$

(ii) Fertilities of matings

$\begin{array}{ll}\text { Mating } & \text { Fertility } \\ A A \times A A & {\left[f u^{2} /(1-t w)+\alpha(1-f) u^{2} /(1-w)\right] / T} \\ A A \times A a & {[2 f u v /(1-t w)+2 \alpha(1-f) u v /(1-w)] / T} \\ A A \times a a & {[f u w(2-t) /(1-t w)+\alpha(1-f) u w /(1-w)+\beta(1-f) u] / T} \\ A a \times A a & {\left[f v^{2} /(1-t w)+\alpha(1-f) v^{2} /(1-w)\right] / T} \\ A a \times a a & {[f v w(2-t) /(1-t w)+\alpha(1-f) v w /(1-w)+\beta(1-f) v] / T} \\ a a \times a a & {\left[f w^{2}(1-t) /(1-t w)+\beta(1-f) w\right] / T} \\ T=f+(1-f)(\alpha+\beta)\end{array}$


frequency, then Case (i) holds initially; if $a$ has recently entered the population, then Case (ii) holds. Case (i) will usually hold if the recessive phenotype has become at least not uncommon in the population.

Case (i). The males mate with frequencies:

\begin{tabular}{ccl} 
& $\begin{array}{c}\text { in preferential } \\
\text { matings }\end{array}$ & \multicolumn{1}{c}{ in random } \\
$A A$ & 0 & $u /(1-t w)$ \\
$A a$ & 0 & $v /(1-t w)$ \\
$a a$ & $\beta$ & $w(1-t) /(1-t w)-\beta$
\end{tabular}

The females, not having been subjected to natural selection, mate with unchanged genotypic frequencies, $u, v$, and $w$. Table 6 shows the different matings, their frequencies and fertilities when there are preferences for both the dominant and recessive phenotypes. When only the recessive is preferred, the recurrence equations are

$$
\begin{aligned}
T u^{\prime} & =f p^{2} /(1-t w) \\
T v^{\prime} & =f(2 p q-t w p) /(1-t w)+\beta(1-f) p \\
T w^{\prime} & =f\left(q^{2}-t w p\right) /(1-t w)+\beta(1-f) q
\end{aligned}
$$

so that

$$
T p^{\prime}=f p\left(1-\frac{1}{2} t w\right) /(1-t w)+\frac{1}{2} \beta(1-f) p
$$

and

$$
T \Delta p=\frac{1}{2} p[-\beta(1-f)+t w(\beta+f-\beta f)] /(1-t w)
$$

where

$$
T=f+\beta(1-f) \text {. }
$$

An equilibrium is therefore established at the point

$$
w_{*}=\frac{\beta(1-f)}{t(\beta+f-\beta f)}
$$

at which the Hardy-Weinberg Law holds. This equilibrium is not the same as that in the corresponding model with polygynous mating in which

$$
w_{*}=\beta / t
$$

By itself, sexual selection gives rise to the same equilibria in both polygynous and monogamous species; the advent of natural selection leads to different equilibria in the two mating systems.

In the present case, the equilibrium represents a polymorphism if

$$
0<\beta<f t /[(1-f)(1-t)]
$$

The rate of approach to $w_{*}$ is determined by the eigenvalue

$$
\lambda=1-p_{*} q_{*} t(\beta+f-\beta f) / f
$$

The equilibrium is clearly stable in the region near $w_{*}$, since the condition for stability, $\beta+f-\beta f>0$, necessarily holds. The corresponding eigenvalue for a polygynous species is given by

$$
\lambda=1-p_{*} q_{*} t /(1-\beta)
$$


This shows that selection is faster in a polygynous species if

and slower if

$$
f<(1-\beta) /(2-\beta)
$$

$$
f>(1-\beta) /(2-\beta)
$$

The recurrence equation for a monogamous species may be written in the alternative form

$$
\Delta p=\frac{1}{2} p t\left(w-w_{*}\right) /(1-t w)
$$

which shows that for all $w>w_{*}, \Delta p>0$ and hence $w_{n+1}<w_{n}$; while for all $w<w_{*}, \Delta p<0$ and hence $w_{n+1}>w_{n}$. The equilibrium is therefore globally as well as locally stable.

Does the condition $\beta \leqq w(1-t) /(1-t w)$ hold at the equilibrium point? It will hold if

$$
\beta \leqq \frac{\beta(1-f)(1-t)}{t(\beta+f-\beta f)} \cdot \frac{\beta+f-\beta f}{f}
$$

or if

$$
f t \leqq(1-f)(1-t)
$$

which is a necessary condition that there may exist a polymorphism.

Case (ii). We now assume that $\beta>w(1-t) /(1-t w)$, which will hold if $a$ is a rare allele that has recently entered the population. If so, then

$$
\begin{aligned}
T u^{\prime} & =f p^{2} /(1-t w) \\
T v^{\prime} & =f(2 p q-t w p) /(1-t w)+p w(1-f)(1-t) /(1-t w) \\
T w^{\prime} & =f\left(q^{2}-t w q\right) /(1-t w)+q w(1-f)(1-t) /(1-t w)
\end{aligned}
$$

so that

$$
T p^{\prime}=f p\left(1-\frac{1}{2} t w\right) /(1-t w)+\frac{1}{2} p w(1-f)(1-t) /(1-t w)
$$

It can then be shown that

$$
T \Delta p=\frac{1}{2} p w[f t-(1-f)(1-t)] /(1-t w)
$$

where

$$
\mathcal{T}=f+w(1-f)(1-t) /(1-t w)
$$

The difference equation shows immediately that if $f t>(1-f)(1-t)$, then $\Delta p>0$ for all values of $p$ and $w$ and hence that $p_{n} \rightarrow 1 \cdot 0$. Since $w$ decreases monotonically as $p$ increases, the initial condition

$$
\beta>w(1-t) /(1-t w)
$$

continues to hold and the recessive phenotype, which is the object of the females' preference, is eliminated. But if $f t<(1-f)(1-t)$, then $\Delta p<0$ and apparently $p_{n} \rightarrow 0$. Before $A$ has been eliminated, however, a point must be reached at which the initial condition for Case (ii) no longer holds. The condition inevitably becomes that for Case (i) at some point during the increase in frequency of $a$. The equilibrium finally reached is therefore given by

$$
w_{*}=\frac{\beta(1-f)}{t(\beta+f-\beta f)}
$$

Thus if we imagine an initial state at which $a$ has just entered the population, 
evolution may take one of two possible directions. If $f t<(1-f)(1-t), a$ moves towards the equilibrium $w_{*}$ at which

so that

$$
w(1-t) /(1-t w)=\beta(1-f)(1-t) / f t
$$

$$
\beta \leqq w(1-t) /(1-t w)
$$

and the condition for Case (i) holds. If $f t>(1-f)(1-t)$, then $a$ is eliminated since fixation of $A$ represents a stable point. On the other hand, if we imagine an initial state at which $A$ has just entered the population, the condition for Case (i) holds: if $\beta<f t /[(1-f)(1-t)]$, a polymorphic equilibrium is established at $w_{*}$; if $\beta \geqq f t /[(1-f)(1-t)], a$ spreads through the population to fixation; $A$ becomes fixed only if $\beta=0$.

The condition $f t>(1-f)(1-t)$ is the same as $f>1-t$ or $t>1-f$. Now $1-f$ is the relative disadvantage suffered by individuals who do not mate preferentially. Near $w=0$, all preferred males mate preferentially since the condition for Case (ii) holds. All the non-preferred individuals are therefore at a relative disadvantage $1-f$. The preferred individuals are all at a disadvantage $t$. Thus if $t>1-f$, preferred individuals have an overall disadvantage and are eliminated. But if $t<1-f$, they have an overall advantage and increase in frequency to reach the polymorphic equilibrium.

(ii) Preference for a deleterious dominant $(\beta=0)$

The evolutionary outcome of selection for a dominant corresponds very closely with that of selection for a recessive.

Case $(i)$. This case, with condition $\alpha \leqq(1-w) /(1-t w)$, leads to recurrence equations as follows, obtained from the fertilities given in table 6 :

giving

$$
\begin{aligned}
T u^{\prime} & =f p^{2} /(1-t w)+\alpha(1-f) p^{2} /(1-w) \\
T v^{\prime} & =f(2 p q-t w p) /(1-t w)+\alpha(1-f)(2 p q-p w) /(1-w) \\
T w^{\prime} & =f\left(q^{2}-t w q\right) /(1-t w)+\alpha(1-f)\left(q^{2}-q w\right) /(1-w)
\end{aligned}
$$

$$
T \Delta p=\frac{1}{2} p w[f t(1-w)+\alpha(1-f)(1-t w)] /[(1-w)(1-t w)]
$$

Since $A$ is now the deleterious phenotype as a result of natural selection, we may put

$$
\begin{aligned}
& t=-s /(1-s) \\
& 1-t w=(1-s+s w) /(1-s)
\end{aligned}
$$

to obtain the recurrence equation

$$
T \Delta p=\frac{1}{2} p w[-f s(1-w)+\alpha(1-f)-\alpha s(1-f)(1-w)] /[(1-w)(1-s+s w)]
$$

Thus $A$ reaches equilibrium at the frequency

$$
1-w_{*}=\frac{\alpha(1-f)}{s(\alpha+f-\alpha f)}
$$

which is equal to the equilibrium frequency of the deleterious recessive when $\alpha=\beta$ and $s=t$. The equilibrium for the dominant is also globally stable like that for the recessive. The rate of approach to $1-w_{*}$ is determined by the eigenvalue

$$
\lambda=1-p_{*} q_{*}[f s-\alpha(1-f)(1-s)][f+\alpha(1-f)] /[f \alpha(1-f)] .
$$


The condition for a polymorphism to exist is

$$
0<\alpha<f s /[(1-f)(1-s)]
$$

which ensures that $\lambda<1$ and the equilibrium is stable.

Case (ii). This case has condition $\alpha>(1-w) /(1-t w)$ and leads to the equations

$$
\begin{aligned}
T u^{\prime} & =p^{2} /(1-t w) \\
T v^{\prime} & =(2 p q-p w) /(1-t w)+f p w(1-t) /(1-t w) \\
T w^{\prime} & =\left(q^{2}-q w\right) /(1-t w)+f q w(1-t) /(1-t w)
\end{aligned}
$$

and hence

$$
T \Delta p=\frac{1}{2} p w(1-f+f t) /(1-t w)
$$

Putting $t=-s /(1-s)$ leads to the equation

$$
T \Delta p=\frac{1}{2} p w(1-s-f) /(1-s+s w) \text {. }
$$

This shows that if $s<1-f, \Delta p>0$ and hence apparently $p_{n} \rightarrow 1 \cdot 0$. However, as $p_{n}$ increases, the point must be reached at which

$$
\alpha \leqq(1-s)(1-w) /(1-s+s w)
$$

and the condition for Case (ii) becomes that for Case (i) giving the equilibrium

$$
1-w_{*}=\frac{\alpha(1-f)}{s(\alpha+f-\alpha f)}
$$

If $s>1-f, p_{n} \rightarrow 0$ and $A$ is eliminated: Case (ii) continues to hold. These results thus parallel those for the recessive in every respect.

\section{Preference For dominant or Recessive PHENOTYPES With NATURAL SELECTION OF BOTH SEXES}

Many characters of sexual display are expressed only in males. As such, and ignoring possible pleiotropic effects on fitness, natural and sexual selection will be limited to males. But many studies of sexual selection have utilised characters that are expressed in both sexes, although choice of mate is restricted to females. Such characters are particular mutants and karyotypes in Drosophila and other insects, and some polymorphic plumage

TABLE 7

Fertilities of the matings with preferences for both dominant and recessive phenotypes

$\begin{array}{ll}\text { Mating } & \text { Fertility } \\ A A \times A A & \left\{f u^{2} /(1-t w)^{2}+\alpha(1-f) u^{2} /[(1-w)(1-t w)]\right\} / T \\ A A \times A a & \left\{2 f u v /(1-t w)^{2}+2 \alpha(1-f) u v /[(1-w)(1-t w)]\right\} / T \\ A A \times a a & \left\{2 f u w(1-t) /(1-t w)^{2}+\alpha(1-f)(1-t) u w /[(1-w)(1-t w)]\right. \\ & \quad+\beta(1-f) u /(1-t w)\} / T \\ A a \times A a & \left\{f v^{2} /(1-t w)^{2}+\alpha(1-f) v^{2} /[(1-w)(1-t w)]\right\} / T \\ A a \times a a & \left\{2 f v w(1-t) /(1-t w)^{2}+\alpha(1-f)(1-t) v w /[(1-w)(1-t w)]\right. \\ & \quad+\beta(1-f) v /(1-t w)\} / T \\ a a \times a a & \left\{f w^{2}(1-t)^{2} /(1-t w)^{2}+\beta(1-f)(1-t) w /(1-t w)\right\} / T \\ & T=f+(1-f)(\alpha+\beta)\end{array}$


phenotypes in birds. The sexual selection of such characters is still limited to males, but the natural selection acts on both sexes. The females mate, like the males, with frequencies that have been altered by the action of natural selection. Table 7 shows the fertilities of the different matings when there are preferences for both the dominant and the recessive phenotypes. The selective coefficient, $t$, measures the advantage of $A$ if positive and $a$ if negative.

\section{(i) Preference for a deleterious recessive $(\alpha=0)$}

At the start of selection, conditions for one of the following two cases must hold: Case (i), in which $\beta \leqq w(1-t) /(1-t w)$; and Case (ii), in which $\beta>w(1-t) /(1-t w)$.

Case $(i)$. From the fertilities given in table 7, we obtain the recurrence equations

$$
\begin{aligned}
T u^{\prime} & =f p^{2} /(1-t w)^{2} \\
T v^{\prime} & =f(2 p q-2 t w p) /(1-t w)^{2}+\beta(1-f) p /(1-t w) \\
T w^{\prime} & =f(q-t w)^{2} /(1-t w)^{2}+\beta(1-f)(q-t w) /(1-t w)
\end{aligned}
$$

and therefore

$$
T p^{\prime}=p\left[f+\frac{1}{2} \beta(1-f)\right] /(1-t w)
$$

giving the difference equation

$$
T \Delta p=p\left[-\frac{1}{2} \beta(1-f)+t w(\beta+f-\beta f)\right] /(1-t w)
$$

and phenotypic frequency at equilibrium

$$
w_{*}=\frac{\frac{1}{2} \beta(1-f)}{t(\beta+f-\beta f)}
$$

The Hardy-Weinberg Law no longer holds at equilibrium, as it does when natural selection acts only on males. In the present case, selection acts on both sexes, but differently on one than on the other: females are naturally selected; males are naturally and sexually selected. In general, the Hardy-Weinberg ratios are never maintained at equilibrium when the sexes are selected differently (O'Donald, 1980). Hardy-Weinberg ratios exist at equilibrium if there is either no selection of one sex, or equal selection of both sexes: then the conditions for the stability of the equilibrium and the rate of approach to it are easily obtained from the gradient matrix; the gradient matrix and non-zero eigenvalues can be given in terms of simple general expressions (O'Donald, 1980). But no simple expressions are obtainable when males are selected differently from females. However, the conditions for the stability of the equilibrium can be determined by direct inspection of the recurrence equation for the gene frequency. This can be written in the form

$$
p^{\prime}=p\left[\frac{f+\frac{1}{2} \beta(1-f)}{f+\beta(1-f)}\right] /(1-t w)
$$

It can then be seen that the condition $p^{\prime}>p$ always holds provided that

$$
w<\frac{\frac{1}{2} \beta(1-f)}{t(\beta+f-\beta f)}=w_{*}
$$


Therefore, if $w>w_{*}, p_{n+1}>p_{n}$ and $w_{n+1}<w_{n}$. By similar reasoning, if $w<w_{*}, w_{n+1}>w_{n}$. This proves that $w_{n} \rightarrow w_{*}$ for all values of $w$. Global stability and convergence has thus been demonstrated. The equilibrium frequency, $w_{*}$, represents a polymorphism if

$$
0<\beta<2 f t /[(1-f)(1-t)]
$$

If $\beta \geqq 2 f t /[(1-f)(1-t)]$ then $w_{n} \rightarrow 1 \cdot 0$. Therefore given some preference for the deleterious recessive, either a polymorphism is established or the preferred character becomes fixed.

Case (ii). In this case, we assume that initially $\beta>w(1-t) /(1-t w)$. This condition must hold if $a$ is a rare allele that has entered the population. Then we get the recurrence equations

$$
\begin{aligned}
T u^{\prime} & =f p^{2} /(1-t w)^{2} \\
T v^{\prime} & =f(2 p q-2 t w p) /(1-t w)^{2}+p w(1-f)(1-t) /(1-t w)^{2} \\
T w & =f(q-t w)^{2} /(1-t w)^{2}+q w(1-f)(1-t) /(1-t w)^{2}
\end{aligned}
$$

and therefore, since

$$
\begin{gathered}
T=f+w(1-f)(1-t) /(1-t w) \\
p^{\prime}=p\left[\frac{f+\frac{1}{2} w(1-f)(1-t) /(1-t w)}{f+w(1-f)(1-t) /(1-t w)}\right] /(1-t w)
\end{gathered}
$$

This suggests that an equilibrium is reached at which

$$
\begin{aligned}
\tilde{w}_{*} & =\frac{f t-\frac{1}{2}(1-f)(1-t)}{t[f t-(1-f)(1-t)]} \\
& =\frac{\frac{1}{2}(1-f-t-f t)}{t(1-f-t)}
\end{aligned}
$$

The recurrence equation may be written in the form of the difference equation

$$
\Delta p=\frac{p w t\left(\tilde{w}_{*}-w\right)[f t-(1-f)(1-t)]}{(1-t w)\{f-w[f t-(1-f)(1-t)]\}}
$$

Thus we see that, provided $f t<(1-f)(1-t)$, then $\Delta p>0$ if $w>\tilde{w}_{*}$ and $\Delta p<0$ if $w<\tilde{w}_{*}$. This shows that there is global convergence to $\tilde{w}_{*}$.

Does the equilibrium point $\tilde{w}_{*}$ represent an equilibrium distinct from that for Case (i)? Consider the point at which

$$
\beta=w(1-t) /(1-t w)
$$

This is the point during the increase in frequency of $a$ at which the conditions for Case (ii) become those for Case (i) and yield the equilibrium $w_{*}$. Substituting the value for $\beta$ into the expression for $w_{*}$, we find

$$
w_{*}=\frac{f t-\frac{1}{2}(1-f)(1-t)}{t[f t-(1-f)(1-t)]}=\tilde{w}_{*}
$$

Thus the equilibrium for Case (ii) is to be found at precisely the point at which the conditions for Case (ii) have changed into those for Case (i). 
The population then moves to the equilibrium $w_{*}$, so that in both cases the final equilibrium is given by

$$
w_{*}=\frac{\frac{1}{2} \beta(1-f)}{t(\beta+f-\beta f)}
$$

(ii) Preference for a deleterious dominant $(\beta=0)$

As usual, selection for a dominant is very similar to selection for a recessive.

Case (i). This case, with condition $\alpha \leqq(1-w) /(1-t w)$, holds if the dominant phenotype has become not uncommon in the population. From table 7 we get recurrence equations

$$
\begin{aligned}
T u^{\prime} & =f p^{2} /(1-t w)^{2}+\alpha(1-f) p^{2} /[(1-w)(1-t w)] \\
T v^{\prime} & =f(2 p q-2 t w p) /(1-t w)^{2}+\alpha(1-f)(2 p q-p w-t w p) /[(1-w)(1-t w)] \\
T w^{\prime} & =f(q-t w)^{2} /(1-t w)^{2}+\alpha(1-f)(q-w)(q-t w) /[(1-w)(1-t w)]
\end{aligned}
$$

where

$$
T=f+\alpha(1-f)
$$

Substituting $-s /(1-s)$ for $t$, where $s$ measures the selective disadvantage of the dominant, we obtain the difference equation

$$
T \Delta p=p w\left[\frac{1}{2} \alpha(1-f)-s(\alpha+f-\alpha f)(1-w)\right] /[(1-w)(1-s+s w)]
$$

so that at equilibrium $A$ is found to occur at the frequency

$$
1-w_{*}=\frac{\frac{1}{2} \alpha(1-f)}{s(\alpha+f-\alpha f)}
$$

Given the same values of the parameters, this is the same phenotypic frequency as that for the recessive. It is easily shown to be globally stable.

Case (ii). This case, with condition $\alpha>(1-w) /(1-t w)$, must hold if $A$ is a rare allele. Consequently, we have recurrence equations

$$
\begin{aligned}
& T u^{\prime}=p^{2} /(1-t w)^{2} \\
& T v^{\prime}=[2 p q-2 t w p-p w(1-f)(1-t)] /(1-t w)^{2}
\end{aligned}
$$

and

where

$$
T p^{\prime}=p\left[1-t w-\frac{1}{2} w(1-f)(1-t)\right] /(1-t w)^{2}
$$

$$
\begin{aligned}
T & =f+(1-f)(1-w) /(1-t w) \\
& =1-(1-f)(1-t) w /(1-t w)
\end{aligned}
$$

Then we obtain the difference equation

$$
T \Delta p=p w\left\{t+\frac{1}{2}(1-f)(1-t)-t w[t+(1-f)(1-t)]\right\} /(1-t w)^{2}
$$

giving the equilibrium

$$
\tilde{w}_{*}=\frac{t+\frac{1}{2}(1-f)(1-t)}{t[t+(1-f)(1-t)]}
$$


Since this represents the case when the dominant is deleterious, we again substitute $-s /(1-s)$ for $t$ and thus obtain

$$
1-w_{*}=\frac{f s-\frac{1}{2}(1-f)(1-s)}{s[f s-(1-f)(1-s)]}
$$

which is the same expression as that for the recessive when $s=t$. This equilibrium represents the point at which

$$
\alpha=(1-w) /(1-t w)
$$

and hence the point at which Case (ii) changes into Case (i). From this point, therefore, the population moves to the equilibrium at which

$$
1-w_{*}=\frac{\frac{1}{2} \alpha(1-f)}{s(\alpha+f-\alpha f)}
$$

\section{Conclusions}

We have seen that when sexual selection alone acts on the males of a population, the evolutionary outcome is usually the same in both monogamous and polygynous species of organisms. The final equilibrium is independent of the reduction in the fertility of later matings that is necessary for sexual selection to occur in monogamous species. Any reduction in fertility, however slight, is sufficient to take the population to the same general equilibrium point. Only when all matings are equally fertile does simple random mating replace sexual selection. But the selection can be very slow if the reduction in fertility is slight. Polygyny usually produces faster selection than monogamy.

If $\alpha$ and $\beta$ are proportions of females who express preferences for dominant and recessive phenotypes $A$ and $a$, then $A$ reaches the stable phenotypic equilibrium frequency

$$
1-w_{*}=\alpha /(\alpha+\beta)
$$

where $w_{*}$ is the equilibrium frequency of $a$. Similarly, if $\alpha, \beta$ and $\gamma$ are the preferences expressed for genotypes $A A, A a$ and $a a$, then the allele $A$ will normally reach equilibrium at the gene frequency

$$
p_{*}=\left(\alpha+\frac{1}{2} \beta\right) /(\alpha+\beta+\gamma) \text {. }
$$

However, in monogamous species with certain values of the parameters $\alpha, \beta$ and $\gamma$, the equilibria attained can depend on initial conditions. If $\alpha \leqq u, \beta \leqq v$ and $\gamma>w$ at the start of selection, these conditions may either change in the course of evolution to the conditions $\alpha \leqq u, \beta \leqq v$ and $\gamma \leqq w$ to give the equilibrium frequency $p_{*}$ as shown above; or if $\alpha>0.4$ and $\gamma$ is small, the initial conditions may continue to hold at the point at which equilibrium is attained. If so, the equilibrium frequency can be found by solving the equation

$$
q_{*}^{3}-q_{*}^{2}+(\alpha+\beta) q_{*}-\frac{1}{2} \beta=0
$$

Table 5 gives some numerical examples of this case. Similar results with alternative equilibria are found if the initial conditions are $\alpha>u, \beta \leqq v$ and $\gamma \leqq w$. 
Natural selection has been introduced into the models of sexual selection for dominant and recessive phenotypes. With the advent of natural selection, the equilibria produced by monogamous and polygynous sexual selection are no longer the same. The reduction in the fertility of the later matings partly determines the equilibrium point in monogamous species. In a polygynous species, if natural selection acts only on males, the equilibrium frequency of a deleterious recessive $a$, which is preferred by a proportion $\beta$ of the females, is given by

$$
w_{*}=\beta / t
$$

where $t$ is the natural selective coefficient expressing the relative disadvantage of $a$. If the natural selection acts on both sexes, then

$$
w_{*}=\frac{1}{2} \beta / t \text {. }
$$

In monogamous species, the corresponding equilibria are given by the following equations: when the natural selection acts only on males

$$
w_{*}=\frac{\beta(1-f)}{t(\beta+f-\beta f)}
$$

and when the natural selection acts on both sexes

$$
w_{*}=\frac{\frac{1}{2} \beta(1-f)}{t(\beta+f-\beta f)}
$$

When all matings have equal fertilities $(f=1)$, then as expected

$$
w_{*}=0 \text {. }
$$

Exactly corresponding results have been obtained for the sexual selection of a deleterious dominant phenotype. Natural and sexual selection are thus balanced at different points in polygynous and monogamous species. Table 8 shows some numerical values of the equilibrium frequencies. When

\begin{tabular}{|c|c|c|c|c|c|c|}
\hline \multirow{2}{*}{$\begin{array}{l}\text { Mating } \\
\text { system }\end{array}$} & & \multicolumn{5}{|c|}{ Values of $\beta$} \\
\hline & & 0.01 & $0 \cdot 03$ & 0.05 & $0 \cdot 07$ & 0.09 \\
\hline Polygyny & & $0 \cdot 1$ & $0 \cdot 3$ & 0.5 & 0.7 & 0.9 \\
\hline Monogamy & $\begin{array}{l}f=0.9 \\
f=0.7 \\
f=0.5 \\
f=0.3 \\
f=0.1\end{array}$ & $\begin{array}{l}0.0111 \\
0.0427 \\
0.0990 \\
0.2280 \\
0.8257\end{array}$ & $\begin{array}{l}0.0332 \\
0.1269 \\
0.2913 \\
0.6542 \\
1.0\end{array}$ & $\begin{array}{l}0 \cdot 0552 \\
0 \cdot 2098 \\
0 \cdot 4762 \\
1 \cdot 0 \\
1 \cdot 0\end{array}$ & $\begin{array}{l}0.0772 \\
0.2913 \\
0.6542 \\
1.0 \\
1.0\end{array}$ & $\begin{array}{l}0.0990 \\
0.3714 \\
0.8257 \\
1.0 \\
1.0\end{array}$ \\
\hline
\end{tabular}
preferential matings have fertilities only moderately greater than random matings $(f=0.9,0.7)$, the equilibrium frequency is lower in monogamous

TABLE 8

Equilibrium frequencies of a preferred phenotype with a selective disadvantage of 10 per cent

These values were calculated using the formulae for selection which acts only on males:

$w_{*}=\beta / t$ (polygyny)

$w_{*}=\beta(1-f) /[t(\beta+f-\beta f)]$ (monogamy) 
species than in polygynous species. When preferential matings have twice the fertility of random matings, the equilibrium frequencies are only slightly lower in monogamous species. The equilibrium frequencies are the same in both species at the value

$$
f=(1-\beta) /(2-\beta)
$$

and rapidly become much higher in monogamous species at larger differences in fertility $(f=0 \cdot 3,0 \cdot 1)$. The preferred phenotype may then reach fixation in monogamous species, while a polymorphism is maintained in a polygynous species.

But large differences in fertility are unlikely to occur in nature, even though differences in reproductive success between early and late matings can be considerable. In the Arctic Skua, pairs breeding in the first week of the breeding season produce an average of 1.67 chicks, while pairs breeding in the fifth and last week produce 0.82 chicks giving them a relative fertility of $f=0.49$ compared to the earliest pairs (O'Donald, 1972a). Both preferential and random matings can take place throughout the breeding season. But more of the preferred males are chosen earlier rather than later in the season: the non-preferred males tend to be left unmated at the start of the season and are only chosen after the females have expressed their preferences. The average difference in fertility is then much less than the difference in fertility of females breeding in the first and last weeks of the season. Suppose, for example, that preferred phenotypes of males are at a low frequency. All or most of the preferential matings take place in the first week. Given the reproductive rates of the Arctic Skua, the average fertility of the random matings that take place over the whole of the breeding season is then $f=0.941$ (O'Donald, 1973a). Unless the majority of females have preferences to express, this value of $f$ actually declines as the preferred males increase in frequency. Values of $f$ are never likely to be as low as $f=0 \cdot 5$. Table 8 thus shows that preferred phenotypes will be maintained at much lower frequencies in monogamous species than in polygynous species. If a balance of sexual and natural selection determines the frequencies of alleles for a sexually advantageous character, polygyny will produce a greater development of the character than monogamy. However, if sexual selection were to act alone, with no opposition from natural selection, the allelic frequencies and development of the character would be the same in both polygynous and monogamous species. The greater development of sexual dimorphism and characters for sexual display, so typical of polygynous species, is therefore evidence that natural selection does act to oppose sexual selection in the evolution of these characters. For example, Clutton-Brock, Harvey and Rudder (1977) found that in primates dimorphism in size is related to polygyny. Monogamous species show little dimorphism: males are only slightly larger than females. Polygynous species are highly dimorphic: males may be twice the size of females. There is some evidence, not significant, that dimorphism increases with the degree of polygyny: the most polygynous species with a high ratio of females to males among mating animals are usually the most dimorphic. The greatest increase in dimorphism, which is highly significant, is between the monogamous and polygynous species. These observations are exactly what we should expect if natural and sexual selection determine the degree of sexual dimorphism in both the monogamous and polygynous groups of species. 


\section{REFERENCES}

CLUTTON-BROCK, T. H., HARVEX, P. H., AND RUDDER, B. 1977. Sexual dimorphism, socionomic sex ratio and body weight in primates. Nature, 269, 797-800.

DARWIN, C. R. 1871. The Descent of Man and Selection in Relation to Sex. John Murray, London.

o'DONALD, P. 1972a. Natural selection of reproductive rates and breeding times and its effect on sexual selection. Amer. Natur., 106, 368-379.

o'DONALD, P. 1972b. Sexual selection by variation in fitness at breeding time. Nature, 237, 349-351.

O'DONALD, P. 1973a. Frequency-dependent sexual selection as a result of variation in fitness at breeding time. Heredity, 30, 351-368.

o'Donald, P. 1973b. Models of sexual and natural selection in polygynous species. Heredity, 31, 145-156.

o'DONALD, P. 1974. Polymorphisms maintained by sexual selection in monogamous species of birds. Heredity, 32, 1-10.

o'Donald, P. 1976. Mating preferences and their genetic effects in models of sexual selection for colour phases of the Arctic Skua. Population Genetics and Ecology, ed. S. Karlin and E. Nevo, pp. 411-430. Academic Press, New York.

o'donald, P. 1979. Genetic Models of Sexual Selection. Cambridge University Press, Cambridge.

o'Donald, P. 1980. A general analysis of genetic models with frequency-dependent mating. Heredity, 44, 309-320.

O'DONALD, P., AND DAVIS, J. W. F. 1977. Mating preferences and sexual selection in the Arctic Skua. III. Estimation of parameters and tests of heterogeneity. Heredity, 39, 121-132. 